\title{
ADMISSION AND POWER CONTROL FOR COGNITIVE RADIO NETWORKS BY SEQUENTIAL GEOMETRIC PROGRAMMING
}

\author{
Emiliano Dall'Anese, Seung-Jun Kim, and Georgios B. Giannakis \\ Dept. of Electrical \& Computer Engr., University of Minnesota, Minneapolis, MN 55455, U.S.A. \\ E-mails: \{emiliano, seungjun, georgios\}@umn.edu
}

\begin{abstract}
In cognitive radio (CR) networks, power control is an effective means to limit the interference caused by the CRs upon the incumbent primary users (PUs) to ensure cohabitation of the two systems. When all CR links can not be active at the same time due to excessive interference, an admission control mechanism is necessary to schedule the CR links. Key to both tasks is accurate knowledge of the CR-to-PU channel gains. However, CRs generally face difficulties in estimating the channel gains very accurately, often due to lack of explicit support from the PU systems. In this work, admission and power control algorithms are developed to account for channel uncertainty through probabilistic interference constraints. Both log-normal shadowing and small-scale fading effects are considered through suitable approximations. The resulting problems can be solved via sequential geometric programming. The admission control is based on solving feasibility problems, whereby CR links violating the interference constraints the most are dropped progressively. The feasible point thus found to initialize the power control iterative solver.
\end{abstract}

Index Terms - Cognitive radios, admission control, power control, channel uncertainty, interference modeling, geometric programming.

\section{INTRODUCTION}

The perceived scarcity of spectrum resources along with the proliferation of new wireless systems have motivated a considerable research effort on dynamic spectrum management techniques. In this context, the hierarchical underlay access model [1,2] envisages an opportunistic and non-intrusive spatial reuse of the frequency bands licensed to primary user (PU) systems by cognitive radio (CR) devices. Provided that "spectrum holes" can be identified via CR sensing [3, 4], power control can enable the coexistence of the two systems by carefully limiting the interference inflicted to the PUs, while maximizing the overall CR network performance.

However, power control in CR networks is challenging owing to certain practical issues. specifically, lack of explicit

This work was supported by NSF grants CCF-0830480, CCF-1016605, ECCS-0824007, ECCS-1002180; and QNRF grant NPRP 09-341-2-128. coordination between the PU system and the CR system, renders it generally difficult for CRs to acquire reliable estimates of the CR-to-PU channel gains. This, in turn, makes it hard to predict the actual interference that would be caused to the PU system. Particularly challenging is to acquire the channels of the PU receivers that do not emit RF energy, which nevertheless have to be protected under the PU-CR hierarchy.

On the other hand, to guarantee a basic quality-of-service for the CR network, minimum signal-to-interference-plusnoise-power ratio (SINR) constraints may be imposed to CR links. Together with stringent PU interference constraints, the overall power control problem often becomes infeasible. Thus, it is important to devise appropriate CR link admission control strategies [5].

Admission and power control strategies for CR networks have been studied mostly under the assumption of perfect CR-to-PU channel knowledge [6, 7, 8]. In view of the underlying difficulties in channel estimation, only statistical knowledge of the CR-to-PU channels is assumed here available through spectrum sensing, or, using recent developed scheme for channel gain cartography [9]. Protection of the PU system is enforced in a probabilistic sense by constraining the interference power experienced by PUs to fall below a tolerable level with a prescribed probability. Since exact computation of the interference probabilities is not tractable, the approximation techniques proposed in our companion work [10] are employed. Specifically, the sum of interfering CR signal powers affected by both shadowing and Nakagamidistributed small-scale fading (on top of path loss effects), is approximated as log-normal through the use of the FentonWilkinson method [11].

Admission control is posed as a combinatorial problem and is often NP hard to solve [12]. Therefore, suboptimal heuristic methods are often employed to select a subset of CR links for which minimum-rate requirements and PU protection constraints can be satisfied [5, 6, 7]. A CR admission strategy is proposed in this paper based on imperfect channel knowledge. A feasibility problem is formulated to check the feasibility of PU interference and SINR constraints. When the given set of CR links cannot be accommodated, solving the feasibility problem explicitly suggests the link that should be dropped through the use of auxiliary variables indicating 
the most severely violated constraint.

The power control and the feasibility problems are nonconvex. However, through a suitable reformulation, their Karush-Kuhn-Tucker (KKT) solutions can be obtained via sequential geometric programming (GP). Therefore, efficient interior-point solvers for GPs can be employed. In particular, the solution obtained from the feasibility problem can initialize the successive GP algorithm for solving the power control problem so that a the CR network performance metric is maximized.

The rest of the paper is organized as follows. In Sec. 2, the system model is described and the optimization problem is formulated. In Sec. 3, the probabilistic interference constraints are approximated for mathematical tractability. The admission control problem and its solution based on sequential GP are described in Sec. 4, while the power control problem is dealt with in Sec. 5. Sec. 6 provides with the results from numerical tests, and Section 7 concludes this paper.

\section{PROBLEM FORMULATION}

Consider a CR network comprising $K$ transmitter-receiver pairs, sharing a frequency band with an incumbent PU system. To make opportunistic spatio-temporal reuse of the licensed spectrum, the CR system employs spectrum sensing algorithms to monitor the PU system activity [3, 4]. Based on the sensing results, power control is performed to limit the interference imposed to the PU system, and thus maximize the overall CR network performance.

Let $\mathbf{x}_{k}$ and $\mathbf{u}_{k}$, for $k \in \mathcal{K}:=\{1, \ldots, K\}$, denote the locations of the $k$-th CR transmitter and receiver, respectively, and $g_{\mathbf{x}_{\mathbf{k}} \rightarrow \mathbf{u}_{\mathbf{k}}}$ the channel gain of link $\mathbf{x}_{k} \rightarrow \mathbf{u}_{k}$. Specifically, $g_{\mathbf{x}_{\mathbf{k}} \rightarrow \mathbf{u}_{\mathbf{k}}}$ can be expressed as [13, Ch. 2]

$$
g_{\mathbf{x}_{\mathbf{k}} \rightarrow \mathbf{u}_{\mathbf{k}}}=g_{k}|| \mathbf{x}_{k}-\mathbf{u}_{k} \|^{-\eta} s_{\mathbf{x}_{\mathbf{k}} \rightarrow \mathbf{u}_{\mathbf{k}}}\left|h_{\mathbf{x}_{\mathbf{k}} \rightarrow \mathbf{u}_{\mathbf{k}}}\right|^{2}
$$

where $g_{k}$ captures the antenna and other propagation gains, $\eta>0$ is the path loss exponent, $s_{\mathbf{x}_{\mathbf{k}} \rightarrow \mathbf{u}_{\mathbf{k}}}$ denotes shadow fading, and $h_{\mathbf{x}_{\mathbf{k}} \rightarrow \mathbf{u}_{\mathbf{k}}}$ is the small-scale fading. Shadowing is modeled as log-normal, and $h_{\mathbf{x}_{\mathbf{k}} \rightarrow \mathbf{u}_{\mathbf{k}}}$ is assumed to be Nakagami- $m$ distributed, with $m \geq 1 / 2$ [13, Ch. 2]. It is also assumed that small-scale fading is independent across different links, and of shadow fading. On the other hand, shadowing may be correlated across links [14].

Let $p_{k} \in\left[0, p_{k}^{\max }\right]$ denote the transmit-power of CR $k$, and define $\mathbf{p}:=\left[p_{1}, \ldots, p_{K}\right]^{T}$. Also, let $\pi_{k}$ denote the received PU signal power as well as other interference measured at the $k$-th $\mathrm{CR}$ receiver. Then, the instantaneous SINR at $\mathrm{CR}$ receiver $k$ can be expressed as

$$
\gamma_{k}:=\frac{p_{k} g_{\mathbf{x}_{\mathbf{k}} \rightarrow \mathbf{u}_{\mathbf{k}}}}{\sum_{k^{\prime} \in \mathcal{K}, k^{\prime} \neq k} p_{k^{\prime}} g_{\mathbf{x}_{\mathbf{k}^{\prime}} \rightarrow \mathbf{u}_{\mathbf{k}}}+\pi_{k}+\sigma_{k}^{2}}, \quad \forall k \in \mathcal{K}
$$

where $\sigma_{k}^{2}$ denotes the receiver noise power.
Let $\left\{\mathbf{r}_{r}\right\}_{r=1}^{R}$ denote either the actual locations of $R$ PU receivers (provided they are available), or candidate positions of the PU receivers that serve as reference points for constraining interference to the PU system. In the latter case, obtaining a reasonable set of such positions is possible using the so-termed power spectral density (PSD) map [3], which reveals "crowded" regions in terms of PU signal presence; or the channel gain map [9], which can provide an estimate of the PU coverage region. For example, the set $\left\{\mathbf{r}_{r}\right\}_{r=1}^{R}$ can be obtained by discretizing the boundaries of the PU coverage region. Supposing incoherent superposition of the CR waveforms [15], the instantaneous interference power experienced by the $r$-th PU receiver is given by

$$
i_{r}:=\sum_{k \in \mathcal{K}} p_{k} g_{\mathbf{x}_{\mathbf{k}} \rightarrow \mathbf{r}_{\mathbf{r}}}, \quad r=1,2, \ldots, R
$$

The resource allocation problem of interest is to maximize the weighted sum-rate of the CR system under constraints on (i) the interference power experienced by the PUs located at $\left\{\mathbf{r}_{r}\right\}_{r=1}^{R}$; and (ii) the minimum SINR required by each CR receiver $k$. The latter requirement prevents CRs from operating in a very low SINR regime, and guarantees basic service rates for the CRs.

Due to the lack of explicit coordination between PU and $\mathrm{CR}$ systems, it is usually challenging for the CRs to acquire accurate estimates of the instantaneous CR-to-PU channel gains [1]. This renders it hard to assess the interference caused to the PU system [cf. (3)], and motivates the statistical knowledge of $\left\{g_{\mathbf{x}_{\mathbf{k}} \rightarrow \mathbf{r}_{\mathbf{r}}}\right\}$ used here instead. To protect ongoing PU transmissions based on uncertain channel information, probabilistic interference constraints are employed. Overall, the transmission power vector $\mathbf{p}$ maximizing the $\mathrm{CR}$ weighted sum-rate without disrupting PU transmissions can be obtained by solving

$$
\begin{aligned}
&(\mathrm{P} 1) \max _{\mathbf{p} \succeq \mathbf{0}} \sum_{k \in \mathcal{K}} w_{k} \log _{2}\left(1+\gamma_{k}(\mathbf{p})\right) \\
& \text { subject to } \quad p_{k} \leq p_{k}^{\max }, \quad k \in \mathcal{K} \\
& \gamma_{k}(\mathbf{p}) \geq \gamma_{k}^{\min }, \quad k \in \mathcal{K} \\
& \operatorname{Pr}\left\{i_{r}>i_{r}^{\max }\right\} \leq \epsilon_{r}, \quad r=1, \ldots, R
\end{aligned}
$$

where $\left\{w_{k}\right\}$ are nonnegative weights, $\gamma_{k}^{\min }$ is the minimum SINR demanded by CR receiver $k$, and $\epsilon_{r}>0$ upper-bounds the probability that the interference due to $\mathrm{CR}$ transmissions exceeds a predetermined threshold $i_{r}^{\max }$ at PU $r$.

Solving (P1) faces a number of challenges. First, a tractable closed-form expression is necessary for the probabilistic constraints (4d). To this end, the approximation method in [10], summarized in Sec. 3, will be employed. Secondly, since (P1) is generally non-convex, it is hard to obtain globally optimal solutions. However, it is possible to reformulate the problem so as to obtain (at least locally) optimal solutions through successive GP $[10,16]$. In order to run 
the successive GP algorithm, one must have available an initial feasible point for (P1). Thus, when impossible to satisfy all the constraints including the individual minimum SINR demands of the CRs, one has to employ an admission control strategy to select the best subset $\mathcal{A} \subseteq \mathcal{K}$ of the $\mathrm{CR}$ links for which (P1) becomes feasible. Since admission control is generally a combinatorial NP hard problem [12], suboptimal heuristics are often employed [5,6]. A CR scheduling scheme will be developed in Sec. 4 based on a feasibility problem formulation, which can also be solved via a sequential GP.

In the sequel, let $A:=|\mathcal{A}| \leq K$ denote the number of admitted CRs, and $\mathbf{p}_{\mathcal{A}}\left(\gamma_{\mathcal{A}}\right)$ stands for the $A \times 1$ vector collecting the transmit-powers $\left\{p_{k}\right\}_{k \in \mathcal{A}}$ (the SINRs $\left\{\gamma_{k}\right\}_{k \in \mathcal{A}}$ ) of the admitted users.

\section{APPROXIMATE INTERFERENCE CONSTRAINTS}

To obtain a tractable expression for the interference constraints (4d), the distribution of interference power $i_{r}=$ $\sum_{k \in \mathcal{A}} p_{k} g_{\mathbf{x}_{k \rightarrow \mathbf{r}_{r}}}$ [cf. (3)] is approximated; see also [10]. Since the random variable (rv) $i_{r}$ involves summation of $A$ powers affected by both shadow fading and small-scale fading, direct characterization of its distribution does not lead to a tractable formulation.

Define $l_{\mathbf{x}_{\mathbf{k}} \rightarrow \mathbf{r}_{\mathbf{r}}}:=s_{\mathbf{x}_{\mathbf{k}} \rightarrow \mathbf{r}_{\mathbf{r}}} \cdot\left|h_{\mathbf{x}_{\mathbf{k}} \rightarrow \mathbf{r}_{\mathbf{r}}}\right|^{2}$ representing the composite fading, as well as its dB-scale version $L_{\mathbf{x}_{\mathbf{k}} \rightarrow \mathbf{r}_{\mathbf{r}}}:=$ $10 \log _{10} l_{\mathbf{x}_{\mathbf{k}} \rightarrow \mathbf{r}_{\mathbf{r}}}$. Let $\mu_{S_{\mathbf{x}_{\mathbf{k}} \rightarrow \mathbf{r}_{\mathbf{r}}}}$ and $\sigma_{S_{\mathbf{x}_{\mathbf{k}} \rightarrow \mathbf{r}_{\mathbf{r}}}}^{2}$ denote the mean and variance of the dB-scale shadowing component $S_{\mathbf{x}_{\mathbf{k}} \rightarrow \mathbf{r}_{\mathbf{r}}}:=$ $10 \log _{10} s_{\mathbf{x}_{\mathbf{k}} \rightarrow \mathbf{r}_{\mathbf{r}}}$. Using these definitions, the probability density function (pdf) of $\mathrm{rv} l_{\mathbf{x}_{\mathbf{k}} \rightarrow \mathbf{r}_{\mathbf{r}}}$ can be well-approximated by the log-normal density as [13, Ch. 2]

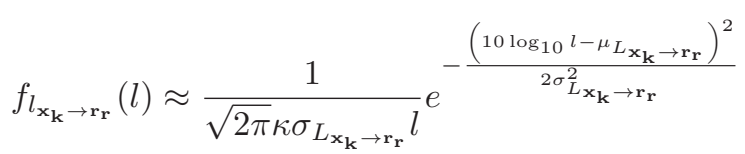

where $\kappa:=\frac{1}{10} \ln 10$ with $\mu_{L_{\mathbf{x}_{\mathbf{k}} \rightarrow \mathbf{r}_{\mathbf{r}}}}$ and $\sigma_{L_{\mathbf{x}_{\mathbf{k}} \rightarrow \mathbf{r}_{\mathbf{r}}}}^{2}$ given by

$$
\begin{aligned}
\mu_{L_{\mathbf{x}_{\mathbf{k}} \rightarrow \mathbf{r}_{\mathbf{r}}}} & :=\frac{1}{\kappa}\left(-\ln m-C+\sum_{m^{\prime}=1}^{m-1} \frac{1}{m^{\prime}}\right)+\mu_{S_{\mathbf{x}_{\mathbf{k}} \rightarrow \mathbf{r}_{\mathbf{r}}}} \\
\sigma_{L_{\mathbf{x}_{\mathbf{k}} \rightarrow \mathbf{r}_{\mathbf{r}}}}^{2} & :=\kappa^{-2} \zeta(2, m)+\sigma_{S_{\mathbf{x}_{\mathbf{k}} \rightarrow \mathbf{r}_{\mathbf{r}}}}^{2}
\end{aligned}
$$

respectively. Here, $C \approx 0.5772$ is Euler's constant and $\zeta(\cdot, \cdot)$ the Hurwitz zeta function. The approximation in (5) is accurate for $\sigma_{S_{\mathbf{x}_{\mathbf{k}} \rightarrow \mathbf{r}_{\mathbf{r}}}} \geq 6 \mathrm{~dB}$ when $m=1$, for $\sigma_{S_{\mathbf{x}_{\mathbf{k}} \rightarrow \mathbf{r}_{\mathbf{r}}}} \geq 4 \mathrm{~dB}$ when $m=2$ and for any $\sigma_{S_{\mathbf{x}_{\mathbf{k}} \rightarrow \mathbf{r}_{\mathbf{r}}}}$ when $m \geq 4$ [13, Ch. 3].

Under (5), the overall channel gain $g_{\mathbf{x}_{\mathbf{k}} \rightarrow \mathbf{r}_{\mathbf{r}}}$ is also approximately log-normal [cf. (1)]. Thus, the rv $i_{r}$ can be viewed as a sum of (possibly correlated) log-normal rv's. Since an exact closed-form expression for the pdf of a sum of log-normally distributed rv's is not available, further approximation is pursued. Specifically, $i_{r}$ will be approximated by another lognormal rv via the Fenton-Wilkinson method $[11,17]$.
To this end, let $G_{\mathbf{x}_{\mathbf{k}} \rightarrow \mathbf{r}_{\mathbf{r}}}:=10 \log _{10} g_{\mathbf{x}_{\mathbf{k}} \rightarrow \mathbf{r}_{\mathbf{r}}}$ denote the Gaussian-approximated channel gain in dB of link $\mathbf{x}_{k} \rightarrow \mathbf{r}_{r}$, and let $\mu_{G_{\mathbf{x}_{\mathbf{k}} \rightarrow \mathbf{r}_{\mathbf{r}}}}:=10 \log _{10}\left(g_{k}\left\|\mathbf{x}_{k}-\mathbf{r}_{r}\right\|^{-\eta}\right)+\mu_{L_{\mathbf{x}_{\mathbf{k}} \rightarrow \mathbf{r}_{\mathbf{r}}}}$ and $\sigma_{G_{\mathbf{x}_{\mathbf{k}} \rightarrow \mathbf{r}_{\mathbf{r}}}}:=\sigma_{L_{\mathbf{x}_{\mathbf{k}} \rightarrow \mathbf{r}_{\mathbf{r}}}}$ be its mean and standard deviation. Let $C_{G_{\mathbf{x}_{\mathbf{k}} \rightarrow \mathbf{r}_{\mathbf{r}}}, G_{\mathbf{x}_{\mathbf{j}} \rightarrow \mathbf{r}_{\mathbf{r}}}}:=\mathbb{E}\left\{\left(G_{\mathbf{x}_{\mathbf{k}} \rightarrow \mathbf{r}_{\mathbf{r}}}-\mu_{G_{\mathbf{x}_{\mathbf{j}} \rightarrow \mathbf{r}_{\mathbf{r}}}}\right)\left(G_{\mathbf{x}_{\mathbf{k}} \rightarrow \mathbf{r}_{\mathbf{r}}}-\right.\right.$ $\left.\left.\mu_{G_{\mathbf{x}_{\mathbf{j}} \rightarrow \mathbf{r}_{\mathbf{r}}}}\right)\right\}$ denote the cross-covariance of $G_{\mathbf{x}_{\mathbf{k}} \rightarrow \mathbf{r}_{\mathbf{r}}}$ and $G_{\mathbf{x}_{\mathbf{j}} \rightarrow \mathbf{r}_{\mathbf{r}}}$ for $(k, r) \neq(j, n)$, and $k, j \in \mathcal{A}$. Upon defining $I_{r}:=$ $10 \log _{10} i_{r}, I_{r}$ can be approximated by a Gaussian rv $\tilde{I}_{r}$ with mean $\mu_{\tilde{I}_{r}}$ and variance $\sigma_{\tilde{I}_{r}}^{2}$ given by $[10,17]$

$$
\mu_{\tilde{I}_{r}}=\kappa^{-1} \ln \left(\frac{\xi_{I_{r}, 1}}{\xi_{I_{r}, 2}^{1 / 2}}\right), \quad \sigma_{\tilde{I}_{r}}^{2}=\kappa^{-2} \ln \left(\frac{\xi_{I_{r}, 2}}{\xi_{I_{r}, 1}}\right)
$$

where

$$
\begin{aligned}
& \xi_{I_{r}, 1}:=\left(\sum_{k=1}^{A} p_{k} a_{r, k}\right)^{2} \\
& \xi_{I_{r}, 2}:=\sum_{k=1}^{A} p_{k}^{2} b_{r, k}+2 \sum_{k=1}^{A-1} \sum_{j=k+1}^{A} p_{k} p_{j} b_{r, k, j}^{\prime} \\
& a_{r, k}:=e^{\kappa \mu_{G_{\mathbf{x}_{\mathbf{k}} \rightarrow \mathbf{r}_{\mathbf{r}}}}+\frac{\kappa^{2}}{2} \sigma_{G_{\mathbf{x}_{\mathbf{k}} \rightarrow \mathbf{r}_{\mathbf{r}}}^{2}}} \\
& b_{r, k}:=e^{2 \kappa \mu_{G_{\mathbf{x}_{\mathbf{k}} \rightarrow \mathbf{r}_{\mathbf{r}}}}+2 \kappa^{2} \sigma_{G_{\mathbf{x}_{\mathbf{k}} \rightarrow \mathbf{r}_{\mathbf{r}}}^{2}}}
\end{aligned}
$$

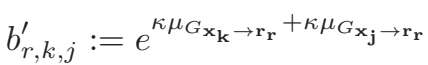

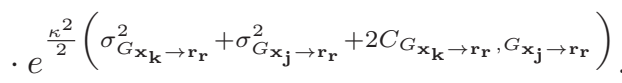

Then, the constraints in (4d) can be replaced by

$$
\begin{gathered}
\operatorname{Pr}\left\{\tilde{I}_{r}\left(\mathbf{p}_{\mathcal{A}}\right)>I_{r}^{\max }\right\} \approx Q\left(\frac{I_{r}^{\max }-\mu_{\tilde{I}_{r}}\left(\mathbf{p}_{\mathcal{A}}\right)}{\sigma_{\tilde{I}_{r}}\left(\mathbf{p}_{\mathcal{A}}\right)}\right) \leq \epsilon_{r}, \\
r=1,2, \ldots, R
\end{gathered}
$$

where $Q(\cdot)$ is the Gaussian tail function, and $I_{r}^{\max }:=$ $10 \log _{10} i_{r}^{\max }$. It follows readily from (14) that

$$
\mu_{\tilde{I}_{r}}\left(\mathbf{p}_{\mathcal{A}}\right)+Q^{-1}\left(\epsilon_{r}\right) \sigma_{\tilde{I}_{r}}\left(\mathbf{p}_{\mathcal{A}}\right) \leq I_{r}^{\max } .
$$

However, plugging (8) into (15) still does not lead to a tractable formulation. This will be possible by introducing a set of positive auxiliary variables $\mathbf{z}_{r}:=\left[z_{r, 1}, z_{r, 2}\right]^{T}$ to arrive at the following set of constraints equivalent to (15):

$$
\begin{gathered}
\frac{\xi_{I_{r}, 1}^{2}\left(\mathbf{p}_{\mathcal{A}}\right)}{\xi_{I_{r}, 2}\left(\mathbf{p}_{\mathcal{A}}\right) z_{r, 1}^{2 \kappa}} \leq 1 \\
\frac{\xi_{I_{r}, 2}\left(\mathbf{p}_{\mathcal{A}}\right)}{\xi_{I_{r}, 1}\left(\mathbf{p}_{\mathcal{A}}\right) z_{r, 2}^{\kappa^{2}}} \leq 1 \\
\phi_{r}\left(\mathbf{z}_{r}\right):=\ln \left(z_{r, 1}\right)+Q^{-1}\left(\epsilon_{r}\right) \ln ^{\frac{1}{2}}\left(z_{r, 2}\right)-I_{r}^{\max } \leq 0 .
\end{gathered}
$$

Constraints (16)-(18) are now compliant with the sequential GP approach that will be employed for solving (P1). 


\section{ADMISSION CONTROL}

Admission control is formulated here as finding the maximal subset $\mathcal{A} \subseteq \mathcal{K}$ of users for which the minimum SINR and PU protection constraints are satisfied. Formally, the following optimization problem is solved to obtain $\mathcal{A}$ :

$$
\begin{aligned}
\max _{\mathbf{p}_{\mathcal{A} \succeq \mathbf{0},\left\{\mathbf{z}_{r} \succeq \mathbf{0}\right\}, \mathcal{A} \subseteq \mathcal{K}}|\mathcal{A}|} \\
\text { subject to } \quad p_{k} \leq p_{k}^{\max }, \quad \forall k \in \mathcal{A} \\
\gamma_{k}\left(\mathbf{p}_{\mathcal{A}}\right) \geq \gamma_{k}^{\min }, \quad \forall k \in \mathcal{A} \\
\frac{\xi_{I_{r}, 1}^{2}\left(\mathbf{p}_{\mathcal{A}}\right)}{\xi_{I_{r}, 2}\left(\mathbf{p}_{\mathcal{A}}\right) z_{r, 1}^{2 \kappa}} \leq 1, \quad r=1, \ldots, R \\
\frac{\xi_{I_{r}, 2}\left(\mathbf{p}_{\mathcal{A}}\right)}{\xi_{I_{r}, 1}\left(\mathbf{p}_{\mathcal{A}}\right) z_{r, 2}^{\kappa^{2}}} \leq 1, \quad r=1, \ldots, R \\
\phi_{r}\left(\mathbf{z}_{r}\right) \leq 0, \quad r=1, \ldots, R
\end{aligned}
$$

where constraints $(19 \mathrm{e})-(19 \mathrm{f})$ replace $(4 \mathrm{~d})$. Problem $(\mathrm{P} 2)$ is known to be NP-hard. Therefore, a suboptimal approach relying on a greedy heuristic is explored for its solution.

Specifically, an admission control algorithm with two components is considered: i) greedy removal of CR links starting from an initial set of candidate users; and ii) feasibility check given a set of admitted users $\mathcal{A}$. In order to check constraint feasibility in (P2) for a given $\mathcal{A}$, the following feasibility problem is solved:

$$
\begin{aligned}
& \min _{\substack{\mathbf{p}_{\mathcal{A}} \succeq \mathbf{0},\left\{\mathbf{z}_{r} \succeq \mathbf{0}\right\} \\
\mathbf{q} \succeq \mathbf{1},\left\{\mathbf{v}_{r} \succeq \mathbf{1}\right\}}} \prod_{k \in \mathcal{A}} q_{k}^{w_{k}} \prod_{r=1}^{R} v_{r, 1} v_{r, 2} v_{r, 3} \\
& \text { subject to } p_{k} \leq p_{k}^{\max }, \forall k \in \mathcal{A} \\
& \frac{\gamma_{k}^{\min }}{\gamma_{k}\left(\mathbf{p}_{\mathcal{A}}\right)} \leq q_{k}, \quad \forall k \in \mathcal{A} \\
& \frac{\xi_{I_{r}, 1}^{2}\left(\mathbf{p}_{\mathcal{A}}\right)}{\xi_{I_{r}, 2}\left(\mathbf{p}_{\mathcal{A}}\right) z_{r, 1}^{2 \kappa}} \leq v_{r, 1}, \quad r=1, \ldots, R \\
& \frac{\xi_{I_{r}, 2}\left(\mathbf{p}_{\mathcal{A}}\right)}{\xi_{I_{r}, 1}\left(\mathbf{p}_{\mathcal{A}}\right) z_{r, 2}^{\kappa^{2}}} \leq v_{r, 2}, \quad r=1, \ldots, R \\
& \frac{\phi_{r}\left(\mathbf{z}_{r}\right)+c_{r}}{c_{r}} \leq v_{r, 3}, \quad r=1, \ldots, R
\end{aligned}
$$

where $\left\{c_{r}\right\}$ are positive constants. If the minimum cost exceeds 1 , the set of users $\mathcal{A}$ is not admissible. Moreover, the optimal variables $\left\{q_{k}^{*}\right\}$ and $\left\{v_{r, 1}^{*}, v_{r, 2}^{*}, v_{r, 3}^{*}\right\}$ explicitly indicate which of the inequalities (19c)-(19f) is violated. If any of the constraints is violated, a least one of the CR links in $\mathcal{A}$ has to be dropped to satisfy it. How to choose which link to remove, is discussed next.

\subsection{Greedy CR link removal}

First, one can quickly shed the links that do not meet the minimum SINR requirement and the PU protection constraint, even if they are scheduled alone. Thus, an initial trial set of admissible users is formed by selecting the links $k \in \mathcal{K}$ satisfying

$$
\gamma_{k}^{\min } \leq \frac{g_{\mathbf{x}_{\mathbf{k}} \rightarrow \mathbf{u}_{\mathbf{k}}}}{\sigma_{k}^{2}} \cdot \min \left[p_{k}^{\max }, \min _{r}\left\{10^{\frac{-\Delta_{k, r}}{10}}\right\}\right]
$$

with $\Delta_{k, r}:=\mu_{G_{\mathbf{x}_{\mathbf{k}} \rightarrow \mathbf{r}_{\mathbf{r}}}}+Q^{-1}\left(\epsilon_{r}\right) \sigma_{G_{\mathbf{x}_{\mathbf{k}} \rightarrow \mathbf{r}_{\mathbf{r}}}}-I_{r}^{\max }$.

Given a set $\mathcal{A},(\mathrm{P} 3)$ is solved to check feasibility. (How to solve (P3) will be discussed in Sec. 4.2.) If the resulting minimum cost is not greater than $1, \mathcal{A}$ is the set of admissible $\mathrm{CR}$ links. Otherwise, at least one $\mathrm{CR}$ link has to be removed from $\mathcal{A}$. Here, A greedy heuristic is proposed next based on the optimal solution $\left\{q_{k}^{*}\right\}$ and $\left\{v_{r, 1}^{*}, v_{r, 2}^{*}, v_{r, 3}^{*}\right\}$ to (P3).

i) If $\prod_{k \in \mathcal{A}} q_{k}^{*}>1$, then the CR link to be removed from $\mathcal{A}$ is the one with the most severely violated SINR constraint; that is,

$$
\bar{k}=\arg \max _{k \in \mathcal{A}} q_{k}^{*} .
$$

Note that the nonnegative weights $\left\{w_{k}\right\}$ prioritize the enforcement of the minimum SINR constraints.

ii) If $\prod_{k \in \mathcal{A}} q_{k}^{*}=1$ but $\prod_{r=1}^{R} v_{r, 1}^{*} v_{r, 2}^{*} v_{r, 3}^{*}>1$, the $\mathrm{CR}$ link dropped is the one that contributes the most to the PU interference; i.e.,

$$
\bar{k}=\arg \max _{k \in \mathcal{A}}\left[\max _{r}\left\{p_{k}^{*} \cdot 10^{\frac{\Delta_{k, r}}{10}}\right\}\right] .
$$

\subsection{Sequential GP-based solution}

Constraints (20c), (20d) and (20e) are expressed as ratios of posynomials, while function $\phi_{r}\left(\mathbf{z}_{r}\right)$ in (20f) is concave. Thus, (P3) is nonconvex and is in general difficult to solve for the global optimum. However, a KKT point of (P3) can be identified using a successive convex approximation method, briefly summarized next [18].

Consider a nonconvex optimization problem

$$
\begin{gathered}
\min _{\mathbf{p} \in \mathcal{P}} f_{0}(\mathbf{p}) \\
\text { subject to } f_{k}(\mathbf{p}) \leq 0, \quad k \in \mathcal{A}
\end{gathered}
$$

where $f_{0}(\mathbf{p})$ is convex and differentiable, while $f_{k}(\mathbf{p})$ is differentiable. Assume also that $\mathcal{F}:=\left\{\mathbf{p} \in \mathcal{P} \mid f_{k}(\mathbf{p}) \leq 0, k \in\right.$ $\mathcal{A}\}$ is compact. The successive convex approximation method solves a sequence of approximating convex problems. Specifically, one solves per iteration $j$

$$
\begin{aligned}
& \min _{\mathbf{p} \in \mathcal{P}} f_{0}(\mathbf{p}) \\
\text { subject to } & \tilde{f}_{k}\left(\mathbf{p} ; \mathbf{p}^{(j)}\right) \leq 0, \quad k \in \mathcal{A}
\end{aligned}
$$

where $\mathbf{p}^{(j)}$ is the optimal solution from the $(j-1)$-st iteration, and $\tilde{f}_{k}\left(\mathbf{p} ; \mathbf{p}^{(j)}\right)$ is a convex surrogate function for $f_{k}(\mathbf{p})$ satisfying the following conditions for each $k \in \mathcal{A}$. 
c1) $f_{k}(\mathbf{p}) \leq \tilde{f}_{k}\left(\mathbf{p} ; \mathbf{p}^{(j)}\right), \quad \forall \mathbf{p} \in \mathcal{F}^{(j)}$

c2) $f_{k}\left(\mathbf{p}^{(j)}\right)=\tilde{f}_{k}\left(\mathbf{p}^{(j)} ; \mathbf{p}^{(j)}\right)$

c3) $\nabla f_{k}\left(\mathbf{p}^{(j)}\right)=\nabla \tilde{f}_{k}\left(\mathbf{p}^{(j)} ; \mathbf{p}^{(j)}\right)$

In the first iteration, it is necessary to have a feasible point $\mathbf{p}^{(0)} \in \mathcal{F}$. If $\mathcal{F}^{(j)}$ is the feasible region of the $j$-th convex problem, the series $\mathbf{p}^{(j)}, j=1,2, \ldots$, converges to the KKT point of the original problem.

To apply the successive convex approximation method to (P3), appropriate surrogate constraints for (20c)-(20f) need to be chosen. First, the ratio-of-posynomial constraints can be replaced by posynomial constraints using the single condensation method [19]. Specifically, consider a constraint

$$
\frac{\sum_{\ell} n_{\ell}\left(\mathbf{p}_{\mathcal{A}}\right)}{\sum_{\ell} d_{\ell}\left(\mathbf{p}_{\mathcal{A}}\right)} \leq 1
$$

where $\left\{n_{\ell}\left(\mathbf{p}_{\mathcal{A}}\right)\right\}$ and $\left\{d_{\ell}\left(\mathbf{p}_{\mathcal{A}}\right)\right\}$ are monomials. Then the denominator can be approximated by its best local monomial approximation around $\mathbf{p}_{\mathcal{A}}^{(j)}$, in the sense of first-order Taylor approximation, to yield

$$
\frac{\sum_{\ell} n_{\ell}\left(\mathbf{p}_{\mathcal{A}}\right)}{\prod_{\ell}\left(\frac{d_{\ell}\left(\mathbf{p}_{\mathcal{A}}\right)}{\alpha_{\ell}}\right)^{\alpha_{\ell}}} \leq 1
$$

where $\alpha_{\ell}:=d_{\ell}\left(\mathbf{p}_{\mathcal{A}}^{(j)}\right) / \sum_{\ell^{\prime}} d_{\ell^{\prime}}\left(\mathbf{p}_{\mathcal{A}}^{(j)}\right)$.

As for the non-convex constraint (20f), note first that $\phi_{r}\left(\mathbf{z}_{r}\right)$ is a concave function in the variable $\mathbf{z}_{r} \succ \mathbf{0}$. Thus, a supporting hyperplane is an upper-bound to this function. Linearly approximating $\phi_{r}\left(\mathbf{z}_{r}\right)$ around $\mathbf{z}_{r}^{(j)}$ yields

$$
\tilde{\phi}_{r}\left(\mathbf{z}_{r} ; \mathbf{z}_{r}^{(j)}\right):=\frac{z_{r, 1}}{z_{r, 1}^{(j)}}+\frac{Q^{-1}\left(\epsilon_{r}\right) z_{r, 2}}{2 z_{r, 2}^{(j)} \sqrt{\ln z_{r, 2}^{(j)}}}-c_{r}\left(\mathbf{z}_{r}^{(j)}\right)
$$

with

$$
c_{r}\left(\mathbf{z}_{r}^{(j)}\right):=1+\frac{Q^{-1}\left(\epsilon_{r}\right)}{2 \sqrt{\ln z_{r, 2}^{(j)}}}-\phi_{r}\left(\mathbf{z}_{r}^{(j)}\right) .
$$

Thus, a surrogate constraint for (20f) is given by

$$
\frac{1}{c_{r}\left(\mathbf{z}_{r}^{(j)}\right)}\left(\tilde{\phi}_{r}\left(\mathbf{z}_{r} ; \mathbf{z}_{r}^{(j)}\right)+c_{r}\left(\mathbf{z}_{r}^{(j)}\right)\right) \leq v_{r, 3}
$$

with $c_{r}=c_{r}\left(\mathbf{z}_{r}^{(j)}\right)$. Note that for $\mathbf{z}_{r}^{(j)} \succ \mathbf{0}$ and $\epsilon_{r}<0.5$, $c_{r}\left(\mathbf{z}_{r}^{(j)}\right)$ is positive, which confirms that (32) is indeed a posynomial constraint.

Thus, after replacing (20c)-(20f) with their surrogates, it is clear that the resulting approximate problem per iteration $j$ is a GP problem. Although GP problems are not convex in their original form, their globally optimal solution can be obtained by a convex reformulation through a log change of variables [20].
Overall, the problem to solve in the $j$-th iteration is given by (P3) with constraints (20c)-(20e) substituted with the corresponding posynomial surrogates in the forms of (29), and (20f) replaced by (32). It is immediately verified that approximations (29) and (32) satisfy conditions c1)-c3).

\section{POWER CONTROL}

Once a maximal feasible subset $\mathcal{A}$ is found, what remains is to adapt the transmit-powers $\mathbf{p}_{\mathcal{A}}$ of the admitted CR links so that the weighted sum-rate of the $\mathrm{CR}$ (sub-)network is maximized. That is, (P1) needs to be solved with $\mathcal{K}$ and $\mathrm{p}$ replaced by $\mathcal{A}$ and $\mathbf{p}_{\mathcal{A}}$, respectively.

Following the sequential GP approach for solving (P1), a surrogate for the cost in (4a) is needed. Note that maximizing the weighted sum-rate $\sum_{k \in \mathcal{A}} w_{k} \log _{2}\left(1+\gamma_{k}\left(\mathbf{p}_{\mathcal{A}}\right)\right)$ is equivalent to minimizing $\prod_{k \in \mathcal{A}}\left(1+\gamma_{k}\left(\mathbf{p}_{\mathcal{A}}\right)\right)^{-w_{k}}$. Thus, upon introducing the $A \times 1$ vector of auxiliary variables $\mathbf{t}_{\mathcal{A}}:=$ $\left\{t_{k}\right\}_{k \in \mathcal{A}} \succeq \mathbf{0}$, as well as a set of extra constraints [cf. (2)]

$$
\begin{aligned}
& \zeta_{k}\left(\mathbf{p}_{\mathcal{A}}, \mathbf{t}_{\mathcal{A}}\right) \\
& :=\frac{\sum_{k^{\prime}, k^{\prime} \neq k} p_{k} g_{\mathbf{x}_{\mathbf{k}^{\prime}} \rightarrow \mathbf{u}_{\mathbf{k}}}+\pi_{k}+\sigma_{k}^{2}}{\left(\sum_{k^{\prime}} p_{k} g_{\mathbf{x}_{\mathbf{k}^{\prime}} \rightarrow \mathbf{u}_{\mathbf{k}}}+\pi_{k}+\sigma_{k}^{2}\right) t_{k}} \leq 1, \quad k \in \mathcal{A}
\end{aligned}
$$

maximizing the weighted sum-rate is equivalent to minimizing

$$
\mathcal{U}\left(\mathbf{t}_{\mathcal{A}}\right):=\prod_{k \in \mathcal{A}} t_{k}^{w_{k}} .
$$

Thus, the power control problem (P1) is equivalent to

$$
\begin{array}{rlrl}
\min _{\substack{\mathbf{p}_{\mathcal{A}} \succeq \mathbf{0} \\
\left\{\mathbf{z}_{r} \succeq \mathbf{0}\right\} \\
\mathbf{t}_{\mathcal{A}} \succeq \mathbf{1}}} \prod_{k \in \mathcal{A}} t_{k}^{w_{k}} & \\
\text { subject to } \quad p_{k_{\mathcal{A}}} \leq p_{k_{\mathcal{A}}}^{\max }, & k \in \mathcal{A} \\
\gamma_{k}^{-1}\left(\mathbf{p}_{\mathcal{A}}\right) \gamma_{k}^{\min } \leq 1, & k \in \mathcal{A} \\
\zeta_{k}\left(\mathbf{p}_{\mathcal{A}}, \mathbf{t}_{\mathcal{A}}\right) \leq 1, & k \in \mathcal{A} \\
\frac{\xi_{I_{r}, 1}^{2}\left(\mathbf{p}_{\mathcal{A}}\right)}{\xi_{I_{r}, 2}\left(\mathbf{p}_{\mathcal{A}}\right) z_{r, 1}^{2 \kappa}} \leq 1, & r=1, \ldots, R \\
\frac{\xi_{I_{r}, 2}\left(\mathbf{p}_{\mathcal{A}}\right)}{\xi_{I_{r}, 1}\left(\mathbf{p}_{\mathcal{A}}\right) z_{r, 2}^{\kappa^{2}}} \leq 1, & & r=1, \ldots, R \\
\phi_{r}\left(\mathbf{z}_{r}\right) & \leq 0, & & r=1, \ldots, R .
\end{array}
$$

The sequential GP approach of Sec. 4.2 can again be employed to obtain (at least locally) optimal solutions to (P4).

In practice, the sequential GP algorithm requires a stopping criterion. Let $\mathbf{t}_{\mathcal{A}}^{(j)}$ denote the optimal solution of the GP problem at iteration $j$. Then, upon defining an error tolerance $v>0$, a simple stopping rule is to check whether condition $\mathcal{U}\left(\mathbf{t}_{\mathcal{A}}^{(j-1)}\right)-\mathcal{U}\left(\mathbf{t}_{\mathcal{A}}^{(j)}\right) \leq v$ is satisfied

The complete $\mathrm{CR}$ admission and power allocation procedure is tabulated in Algorithm 1. 


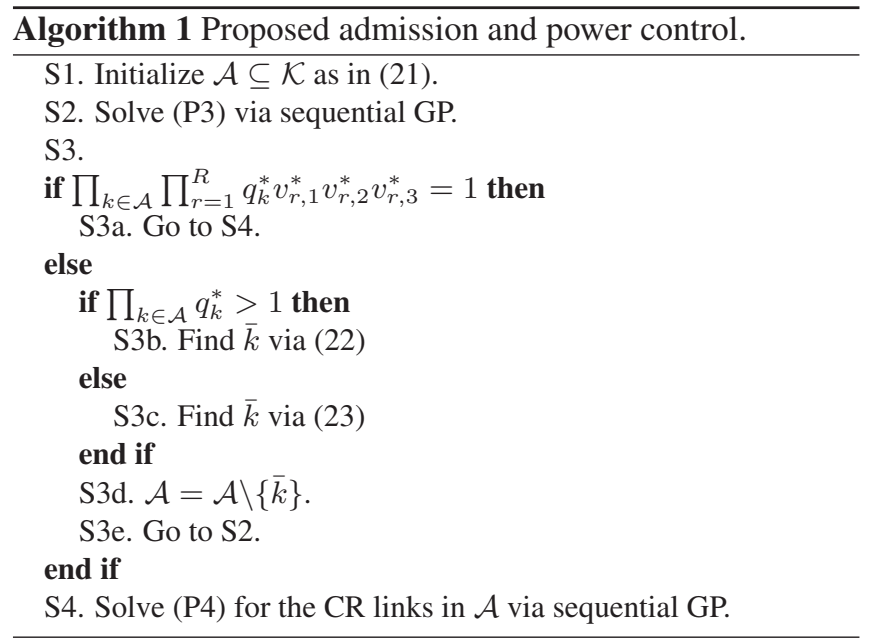

\section{SIMULATIONS}

Consider the scenario depicted in Fig. 1, where $K=5 \mathrm{CR}$ links (shown in solid red lines) are present in an area of $500 \times$ $500 \mathrm{~m}$, and a single $(R=1) \mathrm{PU}$ link (dashed blue line) is also active with transmit-power $1 \mathrm{~W}$. The path loss parameters are set to $g_{k}=1$ for all $k$ and $\eta=3.5$. For Nakagami- $m$ fading, $m=10$ is used. The maximum transmit-power for the CRs is $p_{k}^{\max }=5 \mathrm{~W}$. Spatially correlated log-normal shadowing was generated with mean 0 and coherence distance $30 \mathrm{~m}$, based on the model described in [14]. The interference threshold at the PU receiver was set to $I_{1}^{\max }=-80 \mathrm{dBW}$, and $\epsilon_{1}=0.01$ was used. In order to terminate the sequential GP iterations, a threshold of $v=10^{-4}$ was adopted. Maximizing the sumrate of the CR network with $w_{k}=1$ for all $k$ was considered. The minimum SINRs were fixed to $10 \mathrm{~dB}$ and $0 \mathrm{~dB}$ for CR links $k=1$ and $k=2$, respectively, and to $-5 \mathrm{~dB}$ for links $k=3,4,5$.

To verify the efficacy of the interference constraints, the complementary cumulative distribution function (ccdf) of the received interference power at the PU receiver due to the admitted CRs is plotted in Fig. 2. Different levels of uncertainty were considered by setting the standard deviation of the channel gains to $6 \mathrm{~dB}, 10 \mathrm{~dB}$, and $14 \mathrm{~dB}$ [cf. (7)]. Averaging over 5, 000 independent realizations was used to generate the plot. From Fig. 2, it is clearly seen that Algorithm 1 respects the desired interference constraint. Notice also that larger channel gain uncertainty leads to more conservative power allocation.

Fig. 3 depicts the effect of channel uncertainty on the admission rates (expressed in percentage) of the CR links. The standard deviation of the CR-to-PU channel gains was varied from $6 \mathrm{~dB}$ to $15 \mathrm{~dB}$. As one would expect, larger channel uncertainty yields lower CR link admission rates. The admission rate of link 1 is low due to its stringent SINR requirement. Also, the admission rate for link 2 is significantly lower than those of links 3,4 and 5 .

The influence of the minimum required SINR $\gamma_{k}^{\min }$ on the

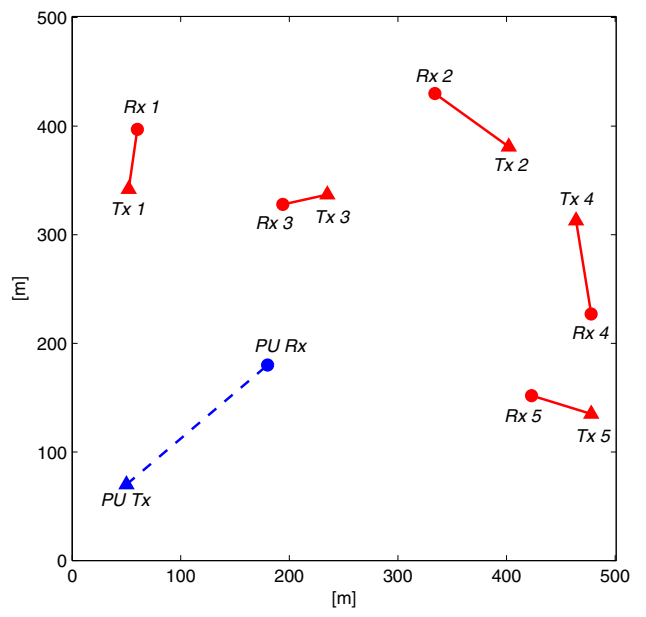

Fig. 1. Test scenario.

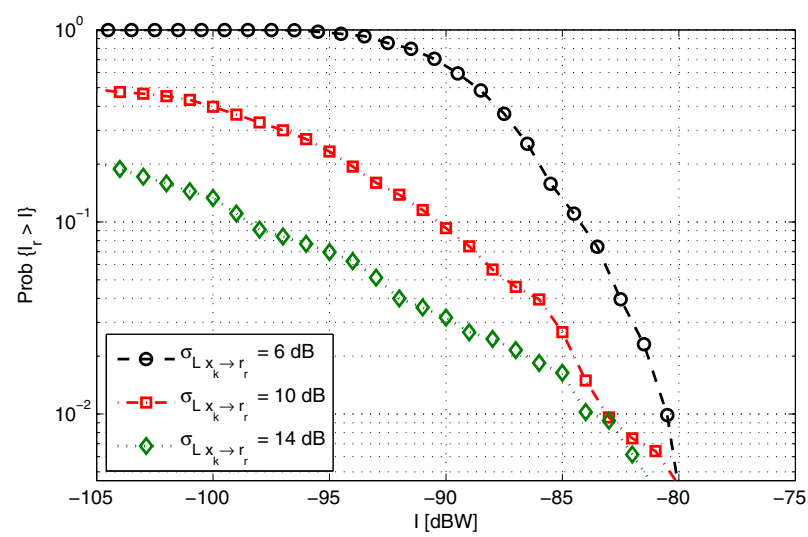

Fig. 2. Interference power ccdf.

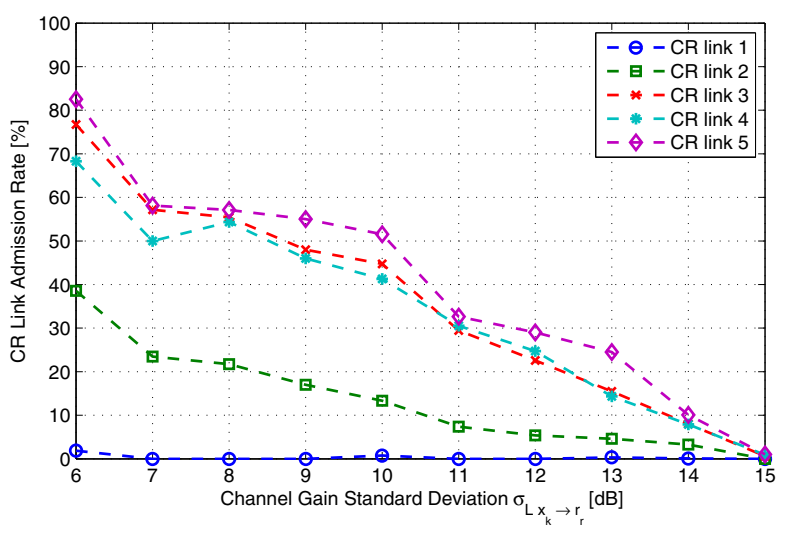

Fig. 3. Admission rates of CR links. 


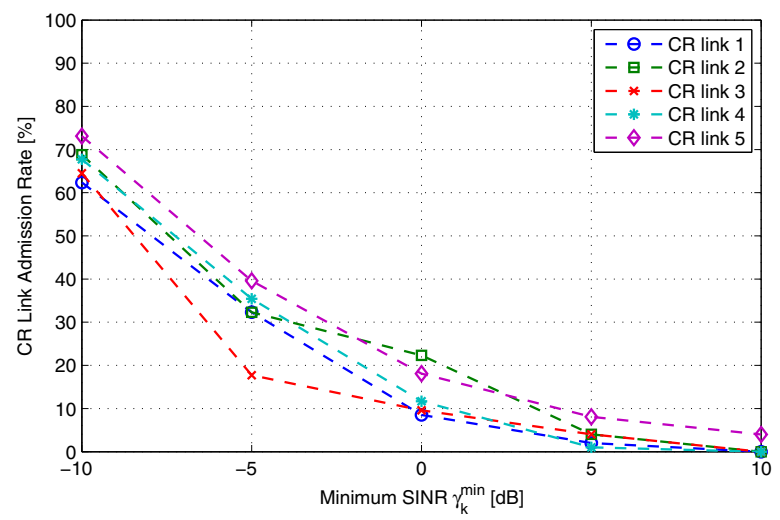

Fig. 4. Link admission rates for different values of $\gamma_{k}^{\min }$.

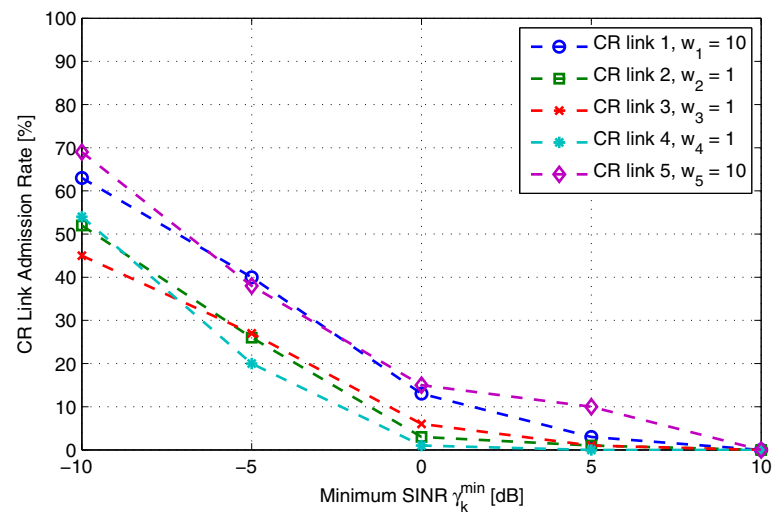

Fig. 5. Admission rates for disparate link weights.

link admission rate is further investigated in Fig. 4. The value of $\gamma_{k}^{\min }$ was set equal for all $k$, and was varied from $-10 \mathrm{~dB}$ to $10 \mathrm{~dB}$. Standard deviation of the channel gain was fixed to $10 \mathrm{~dB}$. It can be seen that the admission rates decrease with increasing $\gamma_{k}^{\min }$.

The effect of the priority weights $\left\{w_{k}\right\}$ is tested in Fig. 5 [cf. (20a)]. Specifically, the weights were set to $w_{1}=w_{5}=$ 10 and $w_{2}=w_{3}=w_{4}=1$. From Fig. 5 it can be observed that CR links 1 and 5 are allowed to transmit more frequently than the others. Thus, properly adjusting the weights can ensure fairness among the links.

\section{CONCLUSIONS}

CR admission and power control algorithms were developed under CR-to-PU channel gain uncertainties arising from shadowing and small-scale fading effects. Probabilistic interference constraints were imposed to protect the PU transmissions notwithstanding the channel uncertainty. The FentonWilkinson method was employed to approximate the distri- bution of the received interference power at the PUs as lognormal, which led to a tractable formulation of the feasibility problem that was solved to form the set of admissible CRs, and the weighted sum-rate maximization problem for the admitted CRs. The admission procedure is based on a gradual link removal strategy, whereby CR links dropped are those violating the interference constraints the most. Due to nonconvexity of the feasibility, and power control problems, a successive convex approximation technique was adopted to obtain KKT optimal solutions. This approach boiled down to a sequential GP algorithm, which can be solved efficiently using, e.g., interior-point methods.

\section{REFERENCES}

[1] Q. Zhao and B. M. Sadler, "A survey of dynamic spectrum access," IEEE Sig. Proc. Mag., vol. 24, no. 3, pp. 79-89, May 2007.

[2] X. Hong, C. Wang, H. Chen, and Y. Zhang, "Secondary spectrum access networks," IEEE Veh. Technol. Mag., vol. 4, no. 2, pp. 36-43, Jun. 2009.

[3] J.-A. Bazerque and G. B. Giannakis, "Distributed spectrum sensing for cognitive radio networks by exploiting sparsity," IEEE Trans. Sig. Proc., vol. 58, no. 3, pp. 1847-1862, Mar. 2010.

[4] S.-J. Kim, E. Dall' Anese, and G.B. Giannakis, "Cooperative spectrum sensing for cognitive radios using Kriged Kalman filtering," IEEE J. Sel. Topics Sig. Proc., vol. 5, no. 1, pp. 24-36, Feb. 2011.

[5] M. Andersin Z. Rosberg and J. Zander, "Gradual removals in cellular PCS with constrained power control and noise," Wireless Networks, vol. 2, no. 1, pp. 27-43, 1996.

[6] I. Mitliagkas, N.D. Sidiropoulos, and A. Swami, "Convex approximation-based joint power and admission control for cognitive underlay networks," in Proc. of the IEEE IWCMC Conf., Crete, Greece, 2008, pp. 28-32.

[7] L. Zhang, Y.-C. Liang, and Y. Xin, "Joint admission control and power allocation for cognitive radio networks," in Proc. of Intl. Conf. on Acoustics, Speech and Signal Proc., Apr. 2007, vol. 3, pp. 673-676.

[8] Y. Xing, C. N. Mathur, M. A. Haleem, R. Chandramouli, and K. P. Subbalakshmi, "Dynamic spectrum access with QoS and interference temperature constraints," IEEE Trans. Mobile Comput., vol. 6, pp. 423-433, Apr. 2007.

[9] E. Dall'Anese, S.-J. Kim, and G.B. Giannakis, “Channel gain map tracking via distributed Kriging," IEEE Trans. Veh. Technol., vol. 60, no. 3, pp. 1205-1211, Mar. 2011. 
[10] E. Dall'Anese, S.-J. Kim, G. B. Giannakis, and S. Pupolin, "Power allocation for cognitive radio networks under channel uncertainty," in Proc. of the Intl. Conf. on Communications, Kyoto, Japan, Jun. 2011 (to appear).

[11] L. F. Fenton, "The sum of lognormal probability distributions in scatter transmission systems," IRE Trans. Commun. Syst., vol. 8, no. 1, pp. 57-67, Mar. 1960.

[12] E. Matskani, N.D. Sidiropoulos, Z.-Q. Luo, and L. Tassiulas, "Convex approximation techniques for joint multiuser downlink beamforming and admission control," IEEE Trans. Wireless Commun., vol. 7, no. 7, pp. 26822693, Jul. 2008.

[13] G. L. Stüber, Principles of Mobile Communication, Kluwer Academic Publishers, Boston, MA, 2nd edition, 2001.

[14] P. Agrawal and N. Patwari, “Correlated link shadow fading in multi-hop wireless networks," IEEE Trans. Wireless Commun., vol. 8, no. 9, pp. 4024-4036, Aug. 2009.

[15] R. Prasad and A. Kegel, "Improved assessment of interference limits in cellular radio performance," IEEE Trans. Veh. Technol., vol. 40, no. 2, pp. 412-419, May 1991.

[16] M. Chiang, C. W. Tan, D. P. Palomar, D. O’Neill, and D. Julian, "Power control by geometric programming," IEEE Trans. Wireless Commun., vol. 6, no. 7, pp. 26402651, Jul. 2007.

[17] A. A. Abu-Dayya and N. C. Beaulieu, "Outage probabilities in the presence of correlated lognormal interferers," IEEE Trans. Veh. Technol., vol. 43, no. 1, pp. 164-173, Feb. 1994.

[18] B. R. Marks and G. P. Wright, "A general inner approximation algorithm for nonconvex mathematical programs," Oper. Res., vol. 26, no. 4, pp. 681-683, Jul.Aug. 1978.

[19] M. Avriel and A. C. Williams, "Complementary geometric programming," SIAM Journal on Applied Mathematics, vol. 19, no. 1, pp. 125-141, Jul. 1970.

[20] S. Boyd and L. Vandenberghe, Convex Optimization, Cambridge University Press, 2004. 